\title{
Increased mitochondrial cytochrome c levels and mitochondrial hyperpolarization precede camptothecin-induced apoptosis in Jurkat cells
}

\author{
JA Sánchez-Alcázar', JG Ault ${ }^{1}$, A Khodjakov ${ }^{1,2}$ and \\ E Schneider ${ }^{*, 1,2}$ \\ 1 Wadsworth Center, New York State Department of Health, Empire State Plaza, \\ Albany, NY 12201, USA \\ 2 Department of Biomedical Sciences, School of Public Health, University at \\ Albany, NY 12201, USA \\ * Corresponding author: E Schneider, Wadsworth Center, Empire State Plaza, \\ Albany, NY 12201 USA. Tel: (518) 474-2088; Fax: (518) 474-1850; \\ E-mail: schneid@wadsworth.org
}

Received 30.3.00; revised 27.6.00; accepted 4.7.00

Edited by $\mathrm{G}$ Salvesen

\begin{abstract}
Mitochondria play a central role in apoptosis through release of cytochrome $\mathrm{c}$ and activation of caspases. In the present study, we showed that, in Jurkat human T cells, camptothecininduced apoptosis is preceded by (i) an increase in cytochrome $c$ and subunit IV of cytochrome $c$ oxidase (COX IV) levels in mitochondria; and (ii) an elevation of the mitochondrial membrane potential $\left(\Delta \Psi_{\mathrm{m}}\right)$. These events are followed by cytochrome $c$ release into the cytosol, cytochrome $\mathrm{c}$ and COX IV depletion from mitochondria, externalization of phosphatidylserine (PS), disruption of $\Delta \Psi_{m}$, caspase activation, poly(ADP-ribose)polymerase cleavage and DNA fragmentation. The pan-caspase inhibitor z-VAD.fmk blocked camptothecin-induced PS externalization, disruption of $\Delta \Psi_{m}$ and DNA fragmentation, suggesting that these events are mediated by caspase activation. In contrast, z-VAD did not prevent cytochromec release, despite preventing cytochrome c and COX IV depletion from mitochondria. Together, these data suggest that mitochondrial cytochrome $c$ and COX IV enrichment are early events preceding the onset of apoptosis and that cytochrome $c$ release is upstream of caspase activation and loss of $\Delta \Psi_{\mathrm{m}}$. Furthermore, prevention by z-VAD of cytochrome $\mathrm{c}$ and COX IV depletion in mitochondria suggests the possibility that a caspase-like activity in mitochondria is involved in the proteolytic depletion of respiratory chain proteins. Activation of this activity may play an important role in drug-induced apoptosis. Cell Death and Differentiation (2000) 7, 1090-1100.
\end{abstract}

Keywords: camptothecin; caspases; cytochrome c; mitochondria; apoptosis

Abbreviations: COX, cytochrome c oxidase; DHFR, dihydrofolate reductase; GAPDH, glyceraldehyde-3-phosphate dehydrogenase; JC-1, 5,5',6,6'-tetrachloro-1,1',3,3'-tetraethylbenzimidazole-carbo- cyanine iodide; MnSOD, manganous superoxide dismutase; PARP, poly(ADP-ribose) polymerase; PI, propidium iodide; VDAC, voltage-dependent anion channel; DIC, difference interference contrast

\section{Introduction}

Apoptosis is a form of programmed cell death that is initiated and completed in an orderly manner and whose purpose is to eliminate the cells without releasing their content into the extracellular environment. ${ }^{1}$ Among the critical cellular mediators of apoptosis are a group of cysteinyl-aspartate proteases, named caspases, ${ }^{2}$ that are normally expressed as latent zymogens, which become activated in response to different proapoptotic stimuli. ${ }^{3-5}$ Following activation, they cleave a variety of cellular proteins such as poly(ADP)polymerase (PARP) ${ }^{6,7}$ Recent data have strongly implicated the mitochondrion as a central integrating organelle in apoptosis, with the capacity to directly activate the execution pathways. In particular, the release of mitochondrial cytochrome $c$ into the cytoplasm is thought to be a critical step in the activation of caspases. ${ }^{8-13}$ Upon entering the cytosol, cytochrome $\mathrm{c}$ becomes associated with a multimeric complex, the apoptosome, comprising cytochrome c, Apaf-1, and caspase-9. Complex formation results in the maturation of caspase-9, which subsequently activates caspase $3 .^{8-10,14}$ Activation of caspase-9 and caspase-3 are central events in the apoptotic process, upon which numerous different signaling pathways converge and through which multiple downstream substrates are cleaved. However, the exact mechanism by which caspases are activated is not clear. Furthermore, the fact that pro-caspase-3 and procaspase- 9 have been found in mitochondria ${ }^{15-17}$ has suggested that activation might also occur in intact mitochondria, which could play a critical role in the initiation of the apoptotic process. ${ }^{18}$

In the present study we investigated the activation of caspase- 9 and caspase- 3 in the cytosol and in the mitochondria from camptothecin-treated Jurkat cells, and the relationship of caspase activation with cytochrome $\mathrm{c}$ release into the cytosol, cytochrome $c$ levels in the mitochondria and mitochondrial membrane potential $\left(\Delta \Psi_{\mathrm{m}}\right)$. We show that camptothecin induced increased mitochondrial cytochrome $\mathrm{c}$ levels and mitochondrial hyperpolarization that precede cytochrome $c$ release and the initiation of the apoptotic program. In addition, we demonstrated that, although the pan-caspase inhibitor zVAD did not prevent cytochrome $c$ release, it nevertheless did prevent the depletion of mitochondrial cytochrome $\mathrm{c}$ and of the intermembrane protein cytochrome c oxidase subunit IV (COX IV) that is seen during apoptosis. This observation 
A

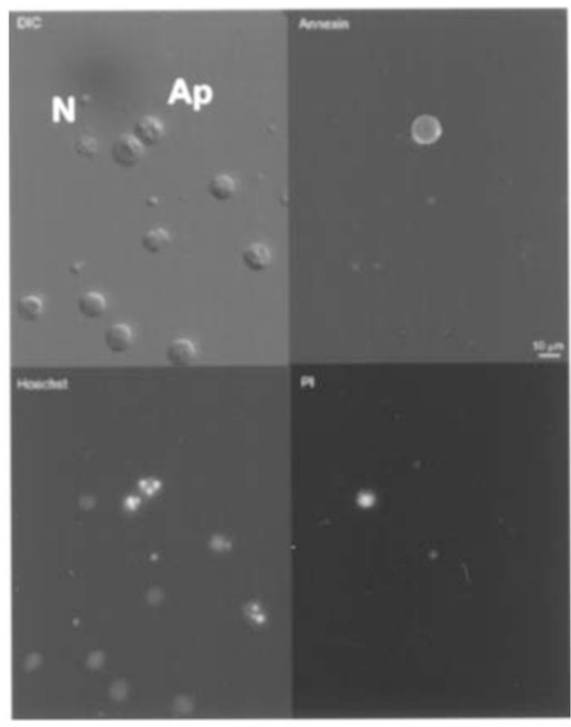

B

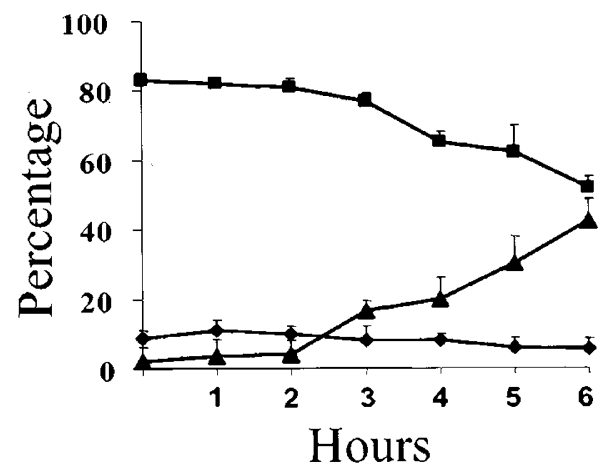

$\underline{\text { SubGo }}$

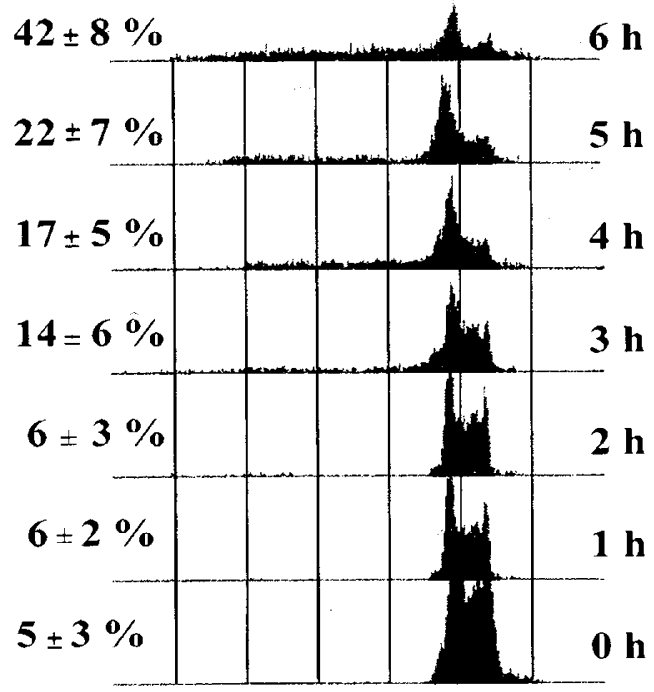

Figure 1 Camptothecin induces apoptosis in Jurkat cells. (A) Jurkat cells were treated with $10 \mu \mathrm{M}$ camptothecin for $6 \mathrm{~h}$. Annexin V binding, Hoechst 33342 and PI staining were performed as described in Materials and Methods. Cells were photographed with a Zeiss fluorescent microscope. N: necrotic; Ap: apoptotic. (B) Time dependent accumulation of necrotic (- -$)$, apoptotic $\left(-\boldsymbol{\Delta}^{-}\right)$and living $(-\mathbf{-}-)$ cells in Jurkat cells as analyzed by bicolor annexin V/ suggested the presence of a caspase-like activity in mitochondria during apoptosis.

\section{Results}

\section{Camptothecin induced apoptosis in Jurkat cells}

To confirm that camptothecin treatment of Jurkat cells induced apoptosis in our hands, several morphological and biochemical tests were performed. First, we examined nuclear morphology and plasma membrane integrity simultaneously by Hoechst and propidium ioidide (PI) double staining. The presence of condensed chromatin without plasma membrane disruption is a main feature of classical apoptosis. As shown in Figure 1A, camptothecin treatment of Jurkat cells resulted in cells with condensed nuclei. Most of the condensed nuclei were $\mathrm{PI}$ negative, indicating that the plasma membrane remained intact. Next, we examined phosphatidylserine (PS) translocation and plasma membrane integrity simultaneously by annexin $\mathrm{V}$ and propidium iodide double staining. In this assay apoptotic cells are positive for annexin $\mathrm{V}$ but negative for PI. We detected an increased number of Annexin $\mathrm{V}+\mathrm{PI}$ cells representing apoptotic cells, concomitant with a decrease in the number of life (Annexin V-/PI-) cells (Figure $1 \mathrm{~B}$ ). The number of necrotic (Annexin $\mathrm{V}+/ \mathrm{Pl}+$ ) cells remained less than $10 \%$ for the entire period of the experiment. Moreover, camptothecin-induced cell death in Jurkat cells was accompanied by a significant increase in the percentage of subG $\mathrm{G}_{0} / \mathrm{G}_{1}$ cells with a subdiploid DNA content indicating DNA degradation (Figure 1C). Together, these observations confirmed that camptothecin treated cells underwent classical apoptosis.

\section{Camptothecin induced increased mitochondrial protein expression before cytochrome $c$ release}

Recent work revealed that cytochrome $c$ is released from the mitochondria during apoptosis. $8,10,19$ To determine the time course of cytochrome $c$ release we treated Jurkat cells with camptothecin $(10 \mu \mathrm{M})$ for various lengths of time, and then separated them into cytosolic and mitochondrial fractions. The presence of cytochrome $c$ in each fraction was analyzed by Western blot using an anti-cytochrome c monoclonal antibody. Starting at $3 \mathrm{~h}$ after the addition of camptothecin, increasing amounts of cytochrome c were detected in the cytosol that continued to increase for several hours (Figure 2A,B). The appearance of cytochrome $\mathrm{c}$ in the cytosol at $3 \mathrm{~h}$ was contemporaneous with a reduction in $\Delta \Psi_{m}$ (Figure $3 \mathrm{~A}$ ), activation of caspase 3 and PARP cleavage (Figure 4), PS translocation (Figure $1 \mathrm{~B}$ ), and DNA fragmentation (Figure 1C). These results confirmed that camptothecin induced all the classical features of apoptosis in our cells. However, the observa-

PI flow cytometric analysis. (C) DNA fragmentation was quantified by measuring the sub $G_{0} / G_{1}$ peak. Cells were treated as in $\mathbf{A}$ for $1,2,3,4,5$ and $6 \mathrm{~h}$. Data represent the means \pm S.D. of three independent experiments 

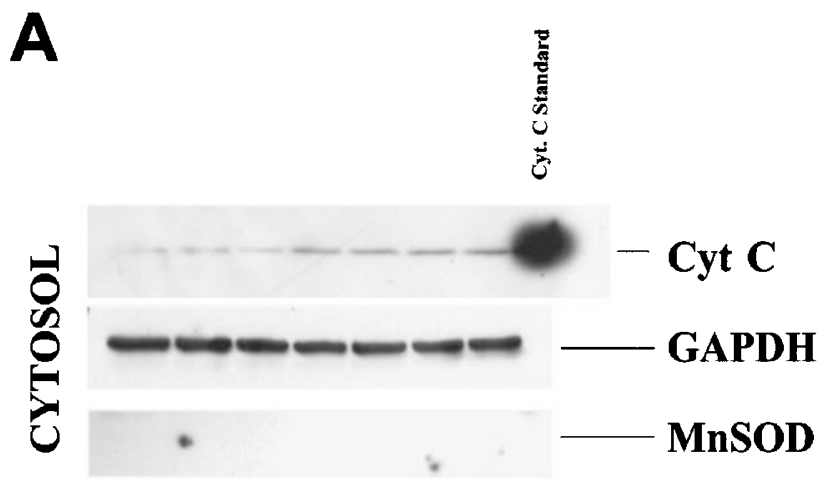

$\begin{array}{llllllll}0 & 1 & 2 & 3 & 4 & 5 & 6 & \text { (hours) }\end{array}$

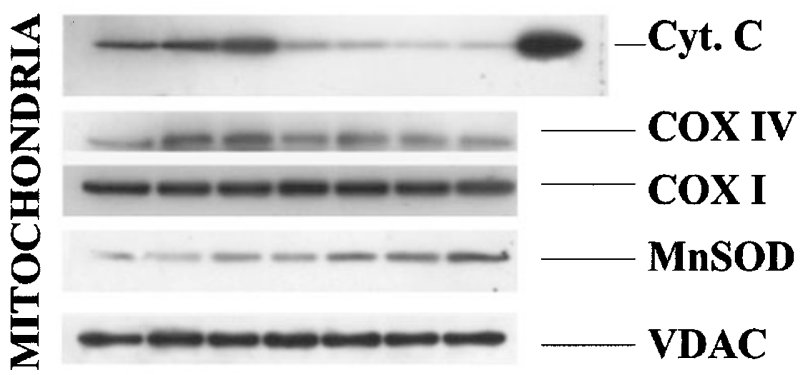

B
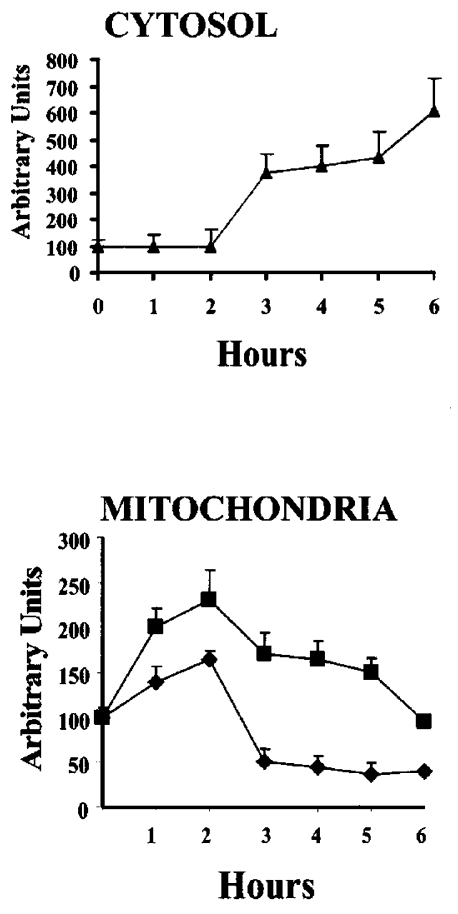

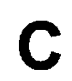

Cytochrome Oxidase Activity

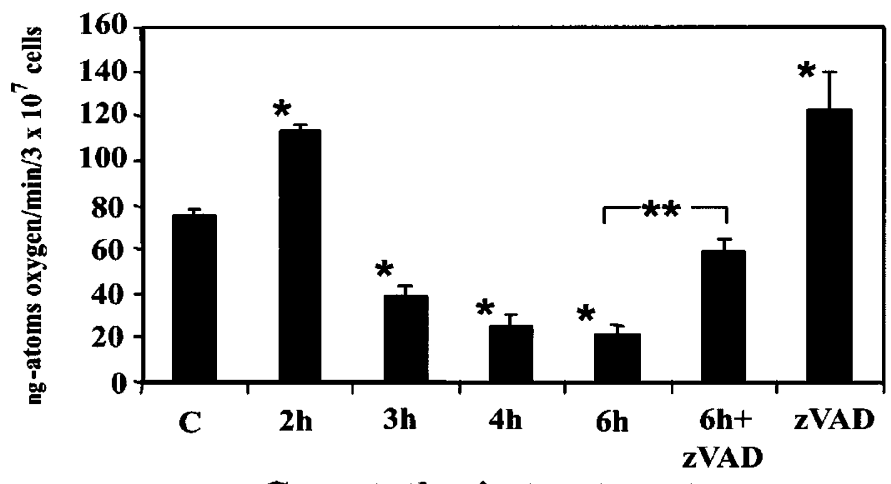

Camptothecin treatment

Figure 2 Effect of camptothecin on the release of cytochrome $\mathrm{c}$ and the expression of mitochondrial proteins. (A) Western blot analysis of cytosolic and mitochondrial extracts from Jurkat cells treated with camptothecin $(10 \mu \mathrm{M})$ for $1,2,3,4,5$, and $6 \mathrm{~h}$. Cytosolic and mitochondrial fractions were prepared as described in Materials and Methods. Membrane blots were incubated with antibodies against cytochrome c, GAPDH, COX IV, COX I, MnSOD and VDAC. The last lane contained $10 \mathrm{ng}$ of horse heart cytochrome $\mathrm{c}$ as a standard. Pictures are representative from one out of three studies. The absence of MnSOD in the cytosolic fraction indicates that there was no mitochondrial contamination. (B) Densitometric quantitation of cytochrome c levels in the cytoplasm (-A-) and of cytochrome $\mathrm{C}$ $(--)$ and COX IV (- -$)$ expression in mitochondria after camptothecin treatment. Results are expressed as means \pm S.D. of three independent experiments. (C) Effect of camptothecin treatment on cytochrome c oxidase-dependent oxygen uptake. Jurkat cells were treated with $10 \mu \mathrm{M}$ camptothecin alone for $2,3,4,5,6 \mathrm{~h}$, and for $6 \mathrm{~h}$ with $10 \mu \mathrm{M}$ camptothecin in the presence or absence of z-VAD $(50 \mu \mathrm{M})$. The cells were harvested and placed in an oxygen electrode cuvette. Oxygen consumption was measured using ascorbate/TMDP as the electron donor. Values are given as means $\pm S$.D. of three independent experiments. ${ }^{\star} P<0.001$ between control and experimental cells. ${ }^{* *} P<0.001$ between the presence and absence of $z$-VAD in camptothecin-treated cells

tion that every event analyzed occurred seemingly at the same time suggested that the onset of the apoptotic process triggers the rapid activation of a cascade of events that are very difficult to order sequentially. 
A

$\underline{\text { Low }} \triangleq \Psi_{\mathrm{m}}$

$39 \pm 5 \%$

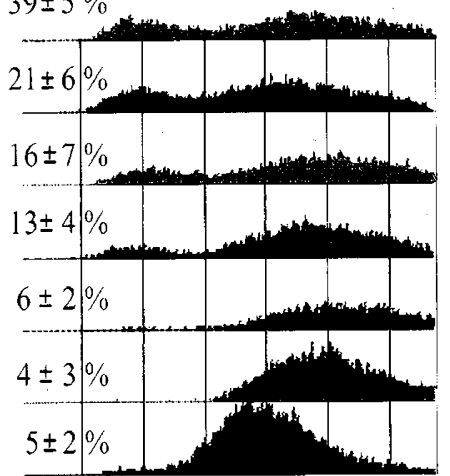

B

Low $\Delta \Psi_{\mathrm{n}}$

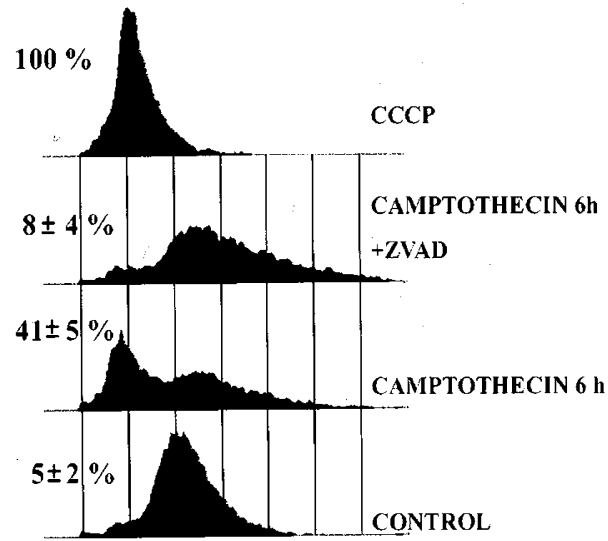

Figure 3 Camptothecin-induced alterations of $\Delta \Psi_{\mathrm{m}}$. (A) Flow cytometric analysis of mitochondrial membrane potential in Jurkat cells treated with camptothecin $(10 \mu \mathrm{M})$ for $0,1,2,3,4,5$ and $6 \mathrm{~h}$. Cells were stained with JC-1 and analyzed by flow cytometry. $n=10000$ cells per analysis. Debris was gated out based on its forward/side scatter (FSC/SSC) characteristics. Individual traces represent histograms of the ratio of red (FL2) versus green (FL1) fluorescence as an estimate of mitochondrial membrane potential $\left(\Delta \Psi_{\mathrm{m}}\right)$. Three separate experiments were conducted which produced results similar to those shown here. (B) Flow cytometric analysis of mitochondrial membrane potential in Jurkat cells treated with camptothecin $(10 \mu \mathrm{M})$ for $6 \mathrm{~h}$ in the absence or presence of $z$-VAD $(50 \mu \mathrm{M})$. As a positive control for reduction of $\Delta \Psi_{\mathrm{m}}$, control cells were treated with the uncoupling agent $\operatorname{CCCP}(1 \mu \mathrm{M})$

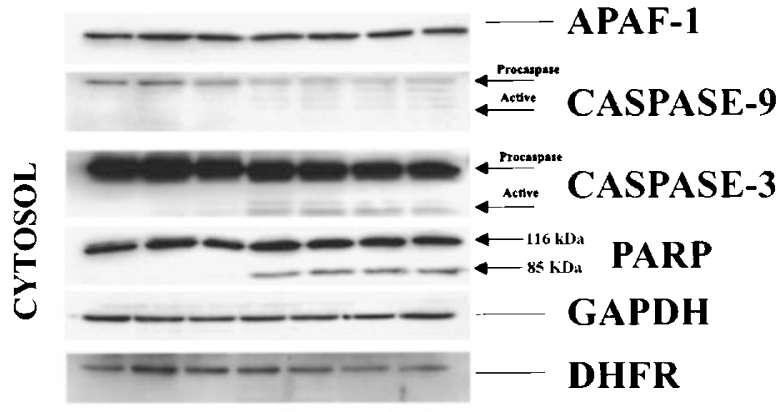

$\begin{array}{llllllll}0 & 1 & 2 & 3 & 4 & 5 & 6 & \text { (hours) }\end{array}$

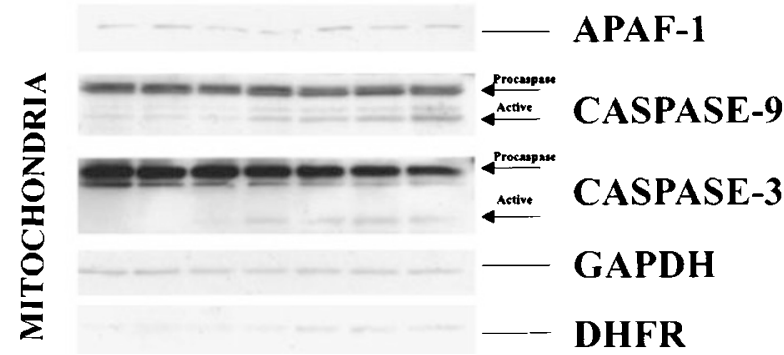

Figure 4 Caspase activation and PARP cleavage. Western blot analysis of cytosolic and mitochondrial extracts from Jurkat cells treated with camptothecin $(10 \mu \mathrm{M})$ for $0,1,2,3,4,5$ and $6 \mathrm{~h}$. The blots were probed with antibodies against caspase-9, caspase-3, Apaf-1 and PARP. GADPH antibodies were used to check equal loading in each lane and DHFR antibodies were used to monitor potential cytosolic contamination of the mitochondrial preparation. The activation of pro-caspase- 9 and pro-caspase-3 is seen as a loss of their pro-forms or as the detection of their active forms (arrows). The PARP cleavage product is indicated by an arrow. Pictures are representative from one out of three studies
The release of cytochrome $c$ into the cytosol is expected to be accompanied by a concomitant decrease in mitochondrial cytochrome c levels. As a control, therefore, we also analyzed cytochrome $c$ levels in the mitochondrial fractions. Surprisingly, this analysis revealed a biphasic alteration in the cytochrome $c$ levels. During the first $2 \mathrm{~h}$ of treatment with camptothecin, the levels of cytochrome $c$ as well as COX IV increased in the mitochondrial fraction (Figure 2A,B). At $3 \mathrm{~h}$, however, coinciding with the beginning of the apoptotic process and the appearance of cytochrome $\mathrm{c}$ in the cytoplasm, there was a substantial drop of cytochrome $\mathrm{c}$ in the mitochondrial fraction to less than $50 \%$ of control levels. Similarly, there was a loss of COX IV, albeit only back to control levels. A small increase over time, but not the subsequent decrease, of the scavenging enzyme MnSOD was also detected. In contrast, we did not detect any changes in either the mitochondrial DNA-encoded subunit I of cytochrome c oxidase or the outer membrane protein VDAC. This suggested that the increase in cytochrome $c$ and COX IV was specific.

To test if the early camptothecin-induced increased expression of some mitochondrial proteins resulted in functional proteins, we examined cytochrome c oxidasedependent oxygen uptake in camptothecin-treated Jurkat cells (Figure 2C). The results showed that concomitant with the higher protein levels there was a significant increase in cytochrome c oxidase activity after $2 \mathrm{~h}$ of treatment. As expected, starting at $3 \mathrm{~h}$ and coinciding with cytochrome $\mathrm{c}$ release and mitochondrial depletion of cytochrome $\mathrm{c}$, cytochrome $\mathrm{c}$ oxidase activity was substantially reduced. These results indicated that the up-regulation of components of the respiratory chain was functional. 


\section{Camptothecin treatment resulted in a transient mitochondrial hyperpolarization}

Loss of mitochondrial membrane potential $\left(\Delta \Psi_{\mathrm{m}}\right)$ has been shown to be an early event during apoptosis in some systems. To determine $\Delta \Psi_{\mathrm{m}}$ we used the membrane potential-sensitive probe $\mathrm{JC}-1$, which forms $\mathrm{J}$-aggregates (with red color) at higher potential and JC-1 monomers (with green color) at low membrane potential, and the ratio between the red and green signals is a measure of $\Delta \Psi_{\mathrm{m}}$. As shown in Figure $3 \mathrm{~A}$, within $1 \mathrm{~h}$ after exposure to camptothecin, there was a shift in the distribution of cells toward a higher red:green ratio, suggesting an increased $\Delta \Psi_{\mathrm{m}}$ in at least some of the cells, which was maintained over the entire incubation period. Based on forward scatter analysis, these cells represent the living population. However, starting at $3 \mathrm{~h}$ an increasing proportion of cells exhibited a decreased $\Delta \Psi_{\mathrm{m}}$. Again, the onset of membrane depolarization coincided with every other endpoint we analyzed. The population with low membrane potential comprises those cells that underwent apoptotic cell death. To confirm that the JC-1 dye was sensitive to mitochondrial membrane depolarization, control Jurkat cells were treated with the mitochondrial uncoupling agent CCCP $(1 \mu \mathrm{M})$. After $10 \mathrm{~min}$ the entire population exhibited a shift in fluorescence from red to green, indicative of a reduction in $\Delta \Psi_{\mathrm{m}}$ (Figure 3B).

\section{Caspase-3 and caspase-9 activation}

Recent studies have demonstrated that the release of cytochrome $\mathrm{c}$ from mitochondria leads to activation of the caspase cascade. ${ }^{11-13}$ In the cytosol, cytochrome $c$ interacts with Apaf-1, which leads to the sequential activation of procaspase- 9 and pro-caspase-3..$^{8,10,14}$ The results in Figure 4
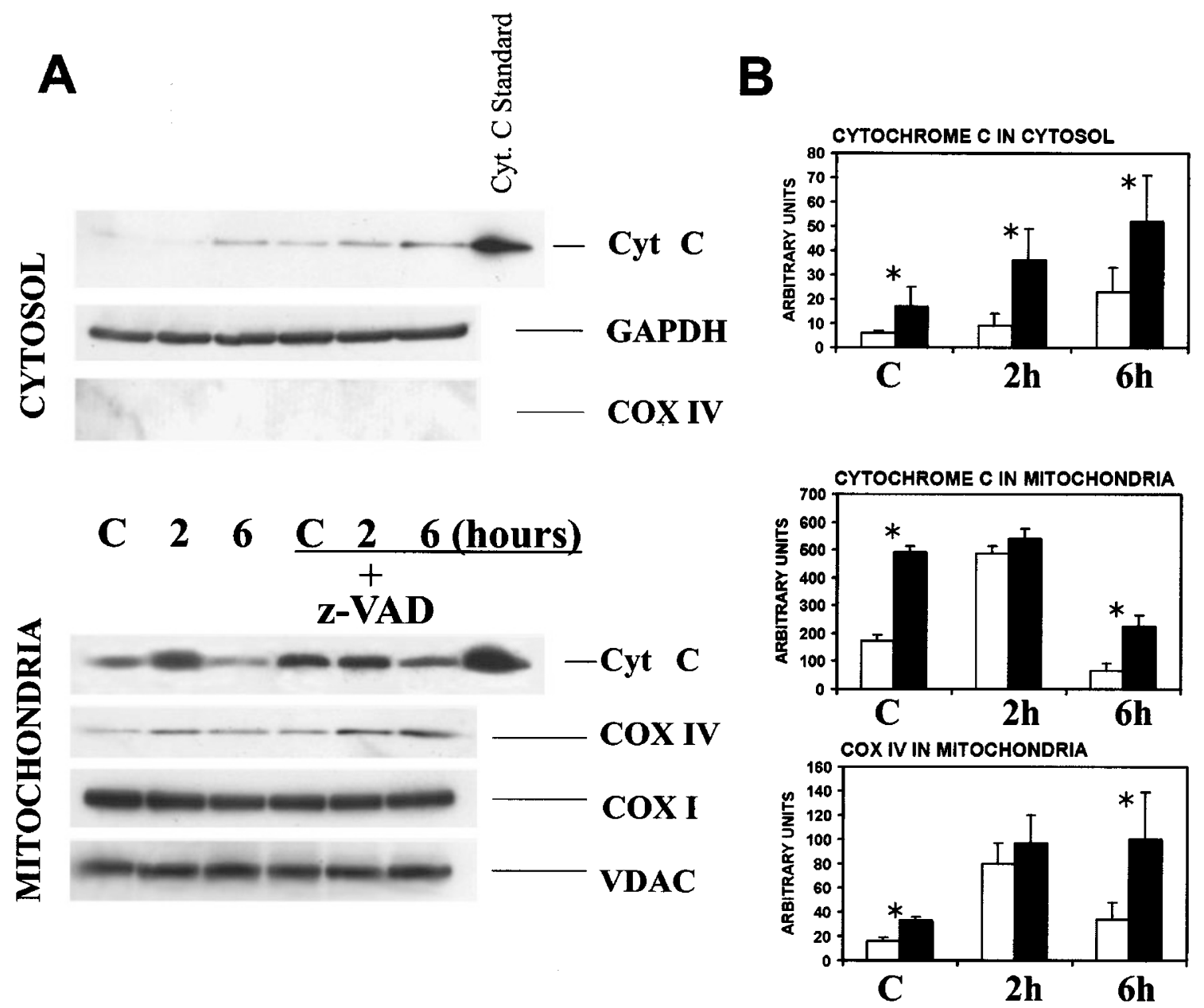

Figure 5 Effect of z-VAD on the release of cytochrome $\mathrm{c}$ and the expression of mitochondrial proteins. (A) Western blot analysis of cytosolic and mitochondrial extracts from Jurkat cells treated with camptothecin $(10 \mu \mathrm{M})$ for 2 and $6 \mathrm{~h}$ in the absence or presence of $\mathrm{z}-\operatorname{VAD}(50 \mu \mathrm{M})$. Control cells were treated for $6 \mathrm{~h}$ with or without Z-VAD alone. Cytosolic and mitochondrial fractions were prepared as described in Materials and Methods. Membrane blots were incubated with antibodies against cytochrome c, GAPDH, COX IV, COX I, and VDAC. The last lane contained $10 \mathrm{ng}$ of horse heart cytochrome $\mathrm{c}$ as a standard. Pictures are representative from one out of three studies. The absence of COX IV in the cytosolic fraction indicates that there was no mitochondrial contamination. (B) Densitometric quantitation of cytochrome $c$ release into the cytoplasm (top panel) and cytochrome $c$ (middle panel) and COX IV (bottom panel) expression in mitochondria after camptothecin treatment in the presence (black bars) or absence (white bars) of $z-V A D(50 \mu M)$. Results are expressed as means $\pm S$.D. of three independent experiments. ${ }^{*} P<0.001$ between the presence and absence of $z-V A D$ 
demonstrate that coinciding with cytochrome $\mathrm{c}$ release into the cytosol at $3 \mathrm{~h}$ there was an activation of both caspase- 9 and caspase- 3 in the cytosolic fraction, which was accompanied by the concomitant cleavage of PARP, as indicated by the appearance of the $M_{r} 85000$ fragment. In contrast, there was no change in cytosolic levels of Apaf-1, GAPDH, and DHFR. Interestingly, when we analyzed the mitochondriacontaining heavy membrane fraction, we observed not only substantial amounts of pro-caspases- 9 and -3 , but also that these caspases were activated at the same time as those in the cytosol. The very low levels of both GAPDH and DHFR indicated that there was minimal cytosolic contamination of this fraction. Thus, these results indicated that substantial amounts of pro-caspases- 9 and -3 were associated with the heavy membrane fraction containing the mitochondria. It remains to be seen, however, if the appearance of the activated caspases was due to direct activation in this fraction, or due to the translocation from the cytoplasm to the heavy membranes after the activation.

\section{Caspase inhibition did not affect cytochrome c release into the cytosol}

To investigate whether there was a connection between the release of cytochrome $\mathrm{c}$, the increase in mitochondrial respiratory chain proteins and caspase activation, Jurkat cells were treated with camptothecin in the presence or absence of the pan-caspase inhibitor z-VAD.fmk. As shown in Figure $5 A, B, z-V A D$ did not prevent the accumulation of cytochrome $\mathrm{c}$ in the cytosol of camptothecin-treated cells, yet effectively inhibited caspase activation and PARP cleavage (Figure 6), loss of $\Delta \Psi_{\mathrm{m}}$ (Figure $3 \mathrm{~B}$ ), PS translocation (Figure $7 A$ ), and DNA fragmentation (Figure 7B). Interestingly, z-VAD by itself also increased cytosolic cytochrome $c$ levels above those seen with camptothecin alone. Furthermore, z-VAD treatment induced an upregulation of cytochrome $\mathrm{c}$ and $\mathrm{COX}$ IV expression in mitochondria and at least partially prevented the camptothecin-induced depletion of these proteins from the organelles. To confirm these results, we also examined cytochrome c oxidase-dependent oxygen uptake in Jurkat cells treated with camptothecin in the presence of $z-V A D$ (Figure 2C). The results showed that in control cells, z-VAD alone induced an increase in cytochrome c oxidase activity, similar to that seen with camptothecin alone after $2 \mathrm{~h}$. Furthermore, z-VAD significantly prevented the loss of cytochrome c oxidase activity seen after $6 \mathrm{~h}$ of camptothecin treatment. Taken together, these observations suggested that z-VAD itself provided a signal to up-regulate some mitochondrial proteins and/or that active caspases may play a role in the depletion (or degradation?) of these proteins in mitochondria during the apoptotic process.

\section{Caspase inhibition prevents mitochondrial degradation}

We had previously observed that in epithelial cells treated with teniposide, mitochondria became either hyperdense or lytic (Sánchez-Alcázar and Schneider, unpublished). In order to investigate whether this was also occurring in Jurkat cells, the cells were treated with camptothecin and analyzed by electron
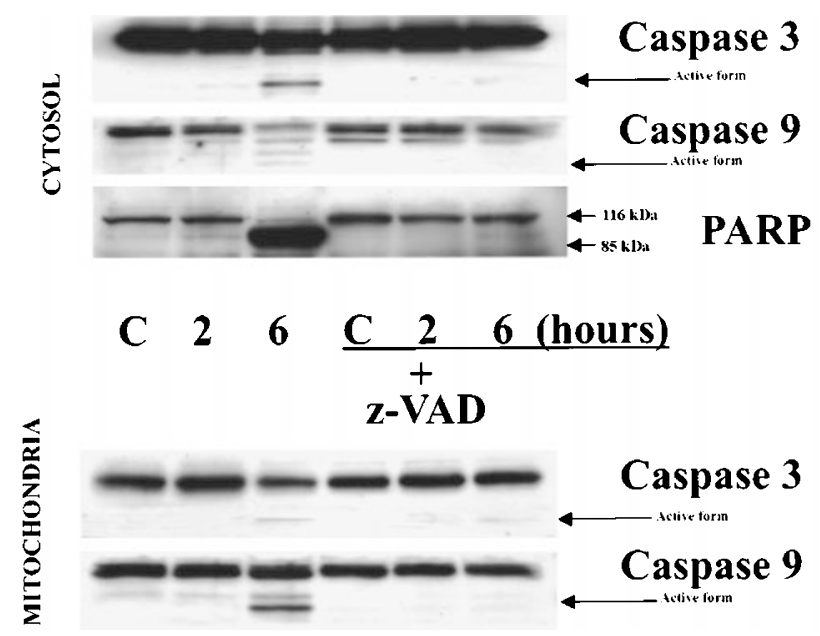

Figure 6 z-VAD blocks caspase activation and PARP cleavage in Jurkat cells. Western blot analysis of cytosolic and mitochondrial extracts from Jurkat cells treated with camptothecin $(10 \mu \mathrm{M})$ for 2 and $6 \mathrm{~h}$ in the presence or absence of $z-\operatorname{VAD}(50 \mu \mathrm{M})$. The blots were probed with antibodies against caspase-9, caspas-3, and PARP. The activation of pro-caspase- 9 and procaspase- 3 is seen as a loss of its pro-form or as the detection of its active forms (arrows). The PARP cleavage product is indicated by an arrow. Pictures are representative from one out of three studies

microscopy (Figure 8). Figure 8A shows an untreated control cell with normal looking mitochondria with regularly stacked cristae. In contrast, Figure 8B shows an apoptotic cell after $6 \mathrm{~h}$ of treatment with camptothecin $(10 \mu \mathrm{M})$. Two types of mitochondria can be seen. The majority of mitochondria look swollen and without the typical cristae structure, but still appear to have a well defined membrane around them (Figure 8B, black arrow), whereas some mitochondria appear morphologically normal (Figure 8B, white arrow). This suggests mitochondrial heterogeneity during the apoptotic process in these cells. The lack of distinct intra-mitochondrial structures in the presence of an apparently intact membrane enclosure suggests that a degradative process has occurred inside these mitochondria. This phenomenon is more clearly seen in Figure 8C,D, which show individual mitochondria from camptothecin-treated cells that look like ghosts surrounded by what appears to be a well defined membrane. In contrast, when cells were treated with camptothecin plus z-VAD $(50 \mu \mathrm{M})$ for $6 \mathrm{~h}$, the mitochondria remained electron dense and looked quite normal (Figure 8E), while the cells displayed condensed chromatin along the nuclear membrane, suggesting that at least some of the nuclear, but not the mitochondrial, events had taken place. Together, these results suggest that during apoptosis in Jurkat cells mitochondria undergo internal z-VAD-sensitive proteolytic degradation while maintaining a surrounding membrane. However, although it appears that the membrane integrity remained preserved, we cannot exclude the possibility that some damage occurred that is not detected by electronmicroscopy.

\section{Discussion}

The present study shows that camptothecin-induced apoptosis of Jurkat cells started at between 2 and $3 \mathrm{~h}$ of 
CONTROL

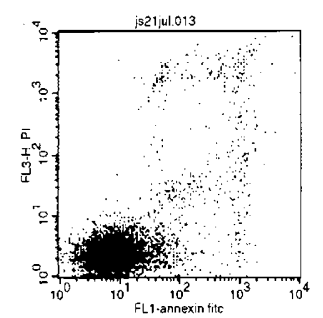

CAMPTOTHECIN $10 \mu \mathrm{M}, 6 \mathrm{H}$

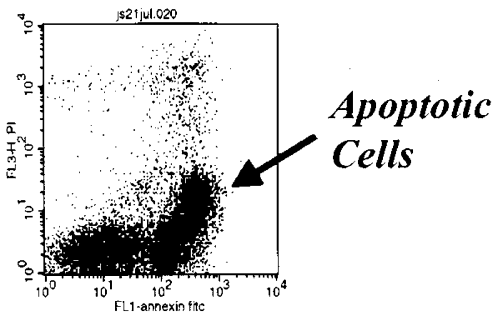

CAMPTOTHECIN $10 \mu \mathrm{M}, 6 \mathrm{H}+$ ZVAD $50 \mu \mathrm{M}$

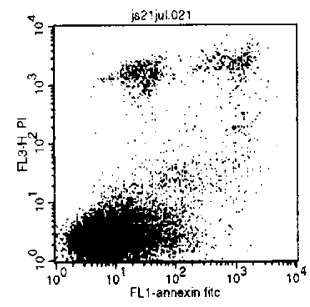

\section{CONTROL}

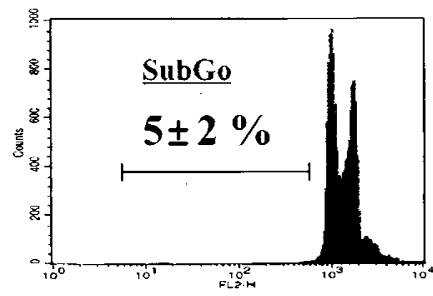

CAMPTOTHECIN $10 \mu \mathrm{M}, 6 \mathrm{H}$

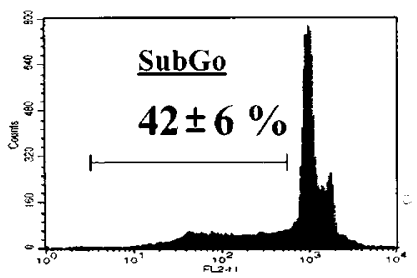

CAMPTOTHECIN $10 \mu \mathrm{M}, 6 \mathrm{H}+\mathrm{ZVAD} 50 \mu \mathrm{M}$

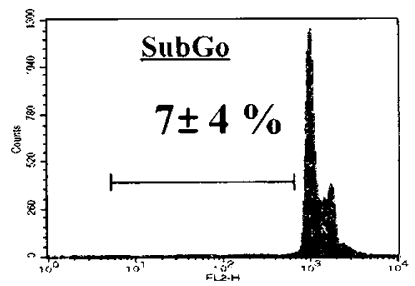

Figure 7 z-VAD inhibits phosphatidylserine translocation and DNA degradation induced by camptothecin. (A) Flow cytometric analysis of annexin $V$ binding (abscissa) versus propidium iodide ( $\mathrm{PI}$ ) uptake (ordinate). Jurkat cells were treated with camptothecin for $6 \mathrm{~h}$ in the presence (bottom panel) or absence (middle panel) of $z-V A D$. Cells were stained with annexin $V$ and $P I$, followed by cytometric analysis. (B) DNA fragmentation was quantified by measuring the sub $\mathrm{G}_{0} / \mathrm{G}_{1}$ peak. Cells were treated as in $\mathbf{A}$ for $6 \mathrm{~h}$ in the presence (bottom panel) or absence (middle panel) of z-VAD (50 $\mu \mathrm{M})$. Data represent the means \pm S.D. of three independent experiments

camptothecin treatment and the number of apoptotic cells gradually increased over the next few hours. In contrast, during the first $2 \mathrm{~h}$ of camptothecin treatment no cell death was detected, which allowed us to examine the immediate response of living cells to the drug treatment and to investigate the early biochemical alterations that precede the onset of apoptosis. These studies demonstrated that camptothecin induced an early increase in mitochondrial levels of cytochrome $\mathrm{c}$ and COX IV and possibly mitochondrial hyperpolarization, that clearly preceded the onset of apoptosis as characterized by PS externalization, caspase activation, loss of $\Delta \Psi_{\mathrm{m}}$, and DNA degradation. Our results are reminiscent of those by other investigators, who concluded that the early increase in $\Delta \Psi_{m}$ was a key event in apoptosis. $^{20-22}$ High levels of respiratory complex proteins have also been observed in the early stages of cell death induced by a novel anti-prostate cancer compound, BMD188, and the authors concluded that active respiration was required for successful apoptosis. ${ }^{22}$ However, the physiolo- gical and/or pathological significance of these initial responses is unknown. Recently, Skulachev has proposed that the role of cytochrome $\mathrm{c}$ in apoptosis might represent one of the anti-oxidant functions inherent in this cytochrome. ${ }^{23}$ First, cytochrome c can operate as an enzyme oxidizing $\mathrm{O}_{2}{ }^{-\bullet}$ back to $\mathrm{O}_{2}$ and the reduced cytochrome $\mathrm{c}$ is then re-oxidized by cytochrome c oxidase. Second, the intermembrane cytochrome $\mathrm{c}$ can activate the electron transport chain in the outer mitochondrial membrane. This bypasses the initial and middle part of the main respiratory chain, which produces, as a rule, the major portion of reactive oxygen species (ROS) in the cell. ${ }^{24,25}$ The fact that camptothecin also up-regulated the mitochondrial expression of MnSOD suggested that free radicals are involved in the pathological changes observed in our experimental system. In this context, up-regulation of cytochrome $\mathrm{c}$ and COX IV levels in mitochondria may play a protective role in the initial response of cells to camptothecin and other potentially cytotoxic insults. Eventually, however, the high levels of cytochrome $\mathrm{c}$ in mitochondria may facilitate 
A

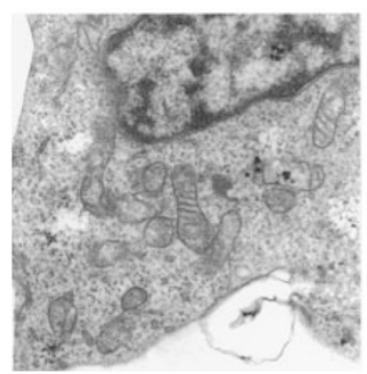

B

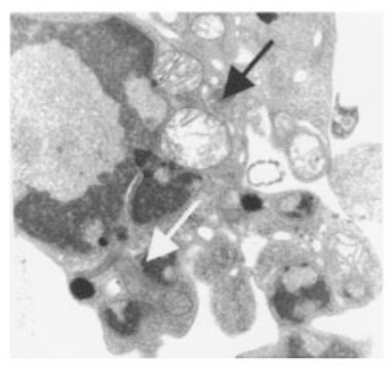

C

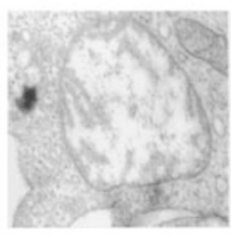

D

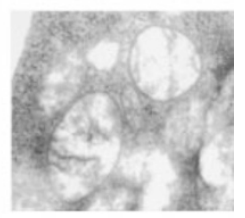

E

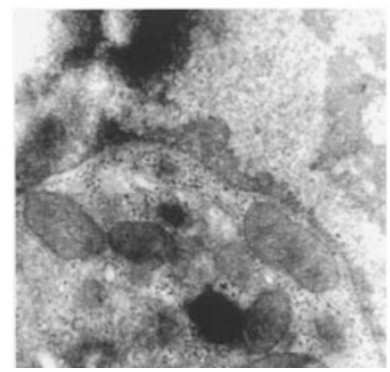

Figure 8 Ultrastructural changes in mitochondria in camptothecin-treated Jurkat cells. (A) Untreated Jurkat cell with a relatively electron-dense cytoplasm and mitochondria with typical, orthodox configuration. $(\mathrm{EM} \times 19000)$. (B) A Jurkat cell treated with $10 \mu \mathrm{M}$ camptothecin for $6 \mathrm{~h}$ showing membrane blebbing, chromatin condensation along the nuclear membrane and a heterogeneous mitochondrial population. The black arrow indicates a mitochondrial ghost with a seemingly intact membrane enclosure, but almost complete lack of internal structure. The white arrow shows a normal looking mitochondrion with the typical cristae structure. $(E M \times 19000)$. (C,D) Individual mitochondria from camptothecin-treated cells. Note the well defined surrounding membrane but almost complete lack of internal structure. $(E M \times 20000)$. (E) Mitochondria in cells treated with camptothecin plus z$\operatorname{VAD}(50 \mu \mathrm{M})$ for $6 \mathrm{~h}$ appear slightly larger but otherwise look normal with a well defined internal morphology. $(\mathrm{EM} \times 25000)$

cytochrome c release (or leakage) and cytosolic caspase activation and eventually apoptotic cell death.

In mitochondria, cytochrome $\mathrm{c}$ is required as an electron carrier in oxidative phosphorylation, and shuttles electrons from Complex III to Complex IV. ${ }^{26}$ The electron transport between these complexes generates a proton gradient across the inner mitochondrial membrane, which maintains $\Delta \Psi_{\mathrm{m}}$. Thus, cytochrome $\mathrm{c}$ release from the electron transport chain is expected to result in impairment of proton flow and a decrease in $\Delta \Psi_{\mathrm{m}}$. The data in the present study support this assumption because the camptothecininduced $\Delta \Psi_{\mathrm{m}}$ drop, starting at $3 \mathrm{~h}$, was accompanied by cytochrome $\mathrm{c}$ release, a depletion of cytochrome $\mathrm{c}$ and COX IV from mitochondria and a reduction in cytochrome $\mathrm{c}$

oxidase activity, caspase activation, and DNA fragmentation. All these events were detected simultaneously after $3 \mathrm{~h}$ of camptothecin treatment, suggesting that once the apoptotic program is initiated, the sequential (or parallel?) cascade of different biochemical and morphological events is very fast and it may be difficult to distinguish the triggering phenomenon from its down-stream consequences.

In an attempt to dissect some of the relationships between these events we blocked caspase activation with the pan-specific inhibitor z-VAD. This effectively prevented known downstream events such as PARP cleavage and DNA fragmentation. Surprisingly, it also blocked the loss of $\Delta \Psi_{\mathrm{m}}$, whereas cytochrome c release was not prevented. This suggested that cytochrome $c$ release was upstream of caspase activation and that $\Delta \Psi_{\mathrm{m}}$ loss was not required for cytochrome $c$ release. Furthermore, it also suggested that loss of $\Delta \Psi_{\mathrm{m}}$ was a consequence of caspase activation and not the cause of it. This interpretation is consistent with data recently reported by Green and coworkers. ${ }^{27,28}$ Similarly, Li et al. recently showed that dissipation of $\Delta \Psi_{\mathrm{m}}$ was not required for caspase activation and even suggested that these two events may be independent of each other. ${ }^{29}$ The fact that even when cytochrome $c$ was released $\Delta \Psi_{\mathrm{m}}$ was maintained has been explained by the import of cytosolic ATP derived from an increased glycolytic rate through the ATP/ADP-translocator. ${ }^{21,30}$ The results in the present study suggest a possible alternative explanation. Even though a substantial amount of cytochrome $\mathrm{c}$ was found in the cytosol in camptothecin plus z-VADtreated cells, the amount remaining in the mitochondria was higher than in camptothecin-only treated cells (Figure 5B). Thus, despite the release of cytochrome $c$ the initially higher levels together with the increased COX IV may provide a sufficient reservoir of these proteins to prevent the decrease of respiratory capacity below a critical threshold level and therefore to maintain the electron flow and $\Delta \Psi_{\mathrm{m}}$.

The exact reasons why z-VAD also prevented mitochondrial cytochrome $c$ depletion remain to be elucidated. One possibility is that mitochondria contain a caspase-like proteolytic activity that is normally responsible for cytochrome $\mathrm{c}$ and COX IV degradation and consequently depletion. In support of this hypothesis our data show the presence of active forms of caspase- 3 and caspase- 9 in the mitochondrial as well as the cytosolic fractions in camptothecin-treated Jurkat cells. Although it is presently not clear if the activation occurred in the cytosol followed by translocation of the activated forms, or if there was activation directly in intact mitochondria, or both, the effect of z-VAD would still be the same. While there is a substantial body of evidence demonstrating that caspases are activated in the cytosol upon formation of the apoptosome complex, direct caspase-9 activation in the mitochondria was recently also proposed by Zhivotovsky and coworkers. ${ }^{18}$ Thus, whatever the mechanism, one can imagine a scenario in which the appearance of active caspases in the mitochondria results in a proteolytic depletion of respiratory chain proteins, which in turn leads to the collapse of $\Delta \Psi_{\mathrm{m}}$ and ultimately to cell death. 
Our observation that z-VAD alone resulted in increased cytochrome $\mathrm{c}$ and COX IV levels in the mitochondria suggests that this inhibitor by itself may be somewhat toxic and may provide a signal to up-regulate mitochondrial respiratory chain protein synthesis, similar to and possibly additive with camptothecin. However, due to its well known effect on the apoptotic caspases it cannot induce the complete death program. Alternatively, it is conceivable that mitochondria contain a $z-V A D$ sensitive constitutive protease that is involved in mitochondrial protein turnover, and whose inhibition results in higher protein levels due to the lack of normal degradation. Consistent with this hypothesis a protease(s) that is associated with the inner membrane has been identified and postulated to play a role in the physiological turnover of mitochondrial proteins and in the formation of respiratory complexes. ${ }^{31-34}$ However, it is not known whether this protease is z-VAD sensitive. Thus, it seems possible that z-VAD could inhibit such a protease and consequently cause higher levels of respiratory chain proteins to accumulate.

In conclusion, our data show that camptothecin induced the up-regulation of cytochrome $\mathrm{c}$ and COX IV in mitochondria, as well as mitochondrial hyperpolarization before any signs of cell death. This may represent the first response of a cell to a potentially toxic insult and be part of a cell's defense mechanism. Eventually, however, the high levels of cytochrome $c$ in mitochondria and mitochondrial hyperpolarization can no longer prevent the progression towards cell death as evidenced by caspase activation, $\Delta \Psi_{\mathrm{m}}$ collapse and cytochrome $\mathrm{c}$ release. The observation that caspase activation and $\Delta \Psi_{\mathrm{m}}$ collapse but not cytochrome $c$ release were prevented by z-VAD suggests the possibility that a caspase(-like) activity in mitochondria may play a crucial role in the apoptotic process.

\section{Materials and Methods}

\section{Reagents}

Camptothecin, horse heart cytochrome c, propidium iodide (PI), and Triton X-100 were purchased from Sigma Chemical Co. (St. Louis, MO, USA). Anti-human cytochrome $\mathrm{c}$ oxidase (subunits I and IV) antibodies were from Molecular Probes (Eugene, OR, USA); antiVDAC antibody was a gift from Dr. CA Mannella (Wandsworth Center, Albany, NY, USA) and anti-MnSOD antibody was a gift from Dr. JA Melendez (Albany Medical College, Albany, NY, USA); anticytochrome $\mathrm{c}$ antibody (clone $7 \mathrm{H} 8.2 \mathrm{C} 12$ ), anti-caspase-3 (CPP32) and anti-caspase- 9 antibodies were from PharMingen (San Diego, CA, USA); anti-PARP antibody was from Roche Biochemicals (Indianapolis, IN, USA); anti-GAPDH and anti-DHFR antibodies were from Research Diagnostic, Inc. (Flanders, NJ, USA). A cocktail of protease inhibitors (complete cocktail) was purchased from Roche Biochemicals (Indianapolis, IN, USA). Z-Val-Ala-Asp-CH2F (z-VAD.fmk) was obtained from Kamiya Biomedical Company (Seattle, WA, USA).

\section{Cell culture}

Jurkat cells were obtained from Dr. D Lawrence (Wadsworth Center, Albany, NY, USA) and grown in RPMI 1640 medium supplemented with penicillin, streptomycin and $10 \% \mathrm{FBS}$ at $37^{\circ} \mathrm{C}$ in a humidified atmosphere of $5 \% \mathrm{CO}_{2}$ in air.

\section{Double staining with Annexin $\mathbf{V}$ and propidium iodide (PI)}

Phosphatidylserine (PS) translocation from the inner to the outer leaflet of the plasma membrane is one of the earliest apoptotic features. We used the PS-binding protein annexin V conjugated with FITC to identify PS exposure in Jurkat cells. The binding of annexin V to cell surface PS was detected with a commercial annexin $\mathrm{V}$ kit (ApoAlert ${ }^{\mathrm{TM}}$ Annexin V-FITC Apoptosis Kit, CLONTECH Laboratories, Palo Alto, CA, USA) according to the manufacturer's instructions. To distinguish cells that had lost membrane integrity, propidium iodide (PI) was added to a final concentration of $10 \mu \mathrm{g} / \mathrm{ml}$ immediately before analysis. Apoptotic cells were identified either by fluorescence microscopy or by flow cytometry with an EPICS Elite flow cytometer (Beckman-Coulter, Hialeah, FL, USA). Hoechst $33342(1 \mu \mathrm{g} / \mathrm{ml})$ staining was used to reveal nuclear condensation.

\section{DNA fragmentation analysis}

DNA fragmentation was measured by quantification of hypoploid nuclei after DNA staining with propidium iodide. Jurkat cells were treated with camptothecin $(10 \mu \mathrm{M})$ for $1,2,3,4,5$, and $6 \mathrm{~h}$. Cells were harvested and fixed with $70 \%$ cold ethanol and left at $4{ }^{\circ} \mathrm{C}$ overnight. Following centrifugation, the fixed cells were resuspended in $1 \mathrm{ml}$ propidium iodide staining solution $(5 \mu \mathrm{g} / \mathrm{ml}$ propidium iodide in $0.1 \%$ sodium citrate, $0.1 \%$ Triton $\mathrm{X}-100)$. The cell suspensions were then incubated for $30 \mathrm{~min}$ at $0^{\circ} \mathrm{C}$. Stained nuclei were analyzed on a FACScan (Becton Dickinson Immunocytometry System, San Jose, CA, USA). Hypoploid cells appear as a $\operatorname{Sub} \mathrm{G}_{0} / \mathrm{G}_{1}$ peak.

\section{Cytochrome c oxidase activity in permeabilized cells}

Cytochrome $\mathrm{c}$ oxidase activity was determined as described. ${ }^{35}$ Jurkat cells were treated with camptothecin $(10 \mu \mathrm{M})$ for $1,2,3,4,5$ and $6 \mathrm{~h}$. Cells were harvested by centrifugation and resuspended in respiration buffer $(0.25 \mathrm{M}$ sucrose, $0.1 \%$ bovine serum albumin, $10 \mathrm{mM} \mathrm{MgCl}$, $10 \mathrm{mM} \mathrm{K}^{+} \mathrm{HEPES}, 5 \mathrm{mM} \mathrm{KH}_{2} \mathrm{PO}_{4}, \mathrm{pH}$ 7.2) at a final concentration of $6 \times 10^{7}$ cells $/ \mathrm{ml}$. One-half $\mathrm{ml}$ of the suspension was injected into a chamber containing $3.5 \mathrm{ml}$ of air-saturated respiration buffer with $1 \mathrm{mM} \mathrm{ADP}$ at $37^{\circ} \mathrm{C}$. The cells were permeabilized with digitonin (final concentration $0.005 \%$ ), and substrates and inhibitors were added in the following order and final concentrations: antimycin A, $50 \mathrm{nM}$; ascorbate, $1 \mathrm{mM}$; TMPD (tetramethyl-p-phenylenediamine), $0.4 \mathrm{mM}$. Antimycin A was used to inhibit autologous mitochondrial electron transport. TMPD is an electron donor that reduces cytochrome $c$ nonenzymatically. Ascorbate was used to reduce TMPD. The oxygen concentration was calibrated with air-saturated buffer, assuming $390 \mathrm{ng}$-atoms of oxygen $/ \mathrm{ml}$ of buffer. Rates of potassium cyanidesensitive oxygen consumption are expressed as ng-atoms of oxygen/ $\mathrm{min} / 3 \times 10^{7}$ cells.

\section{Western blot analysis of cytosolic and mitochondrial fractions}

Cytosolic and mitochondrial fractions were prepared as described. ${ }^{36}$ Jurkat cells were collected by centrifugation at $200 \times g$ for $5 \mathrm{~min}$ at $4^{\circ} \mathrm{C}$. The cells were then washed twice with ice-cold PBS, $\mathrm{pH} 7.4$, followed by centrifugation at $200 \times g$ for $5 \mathrm{~min}$. The cell pellet was resuspended in $600 \mu \mathrm{l}$ of extraction buffer, containing $220 \mathrm{mM}$ mannitol, $68 \mathrm{mM}$ sucrose, $50 \mathrm{mM}$ PIPES- $\mathrm{KOH}, \mathrm{pH} 7.4,50 \mathrm{mM} \mathrm{KCl}$, $5 \mathrm{mM}$ EGTA, $2 \mathrm{mM} \mathrm{MgCl}$, $1 \mathrm{mM}$ dithiothreitol (DTT) and protease inhibitors. After $30 \mathrm{~min}$ incubation on ice, the cells were homogenized 
with a teflon homogenizer for $3 \mathrm{~min}$ at 300 r.p.m. Cell homogenates were centrifuged at $14000 \times g$ for $15 \mathrm{~min}$ and supernatants were removed and stored at $-70^{\circ} \mathrm{C}$. The quality of the cytosolic and mitochondrial fractions was routinely monitored by Western blotting for cytochrome oxidase subunit I as a marker for mitochondrial contamination of the cytosol and for DHFR and GAPDH as markers for cytosolic contamination of the mitochondrial fraction. Twenty-five $\mu \mathrm{g}$ of cytosolic proteins were separated on a $15 \%$ denaturing SDSPAGE minigel. After protein transfer, the membrane was incubated with various primary antibodies. Anti-cytochrome $c$, anti-cytochrome $c$ oxidase subunits I and IV, anti-PARP, anti-caspase-3 and anticaspase-9, anti-VDAC, and anti-DHFR antibodies were diluted $1: 1000$. Anti-GAPDH monoclonal antibody was diluted $1: 5000$. The membrane was then incubated with the corresponding secondary antibody coupled to horseradish peroxidase at 1:10 000 dilution. The specific protein complexes were identified using the 'Supersignal' substrate chemiluminescence reagent (Pierce, Rockford, IL, USA).

\section{Measurement of mitochondrial membrane potential by flow cytometry}

Changes in the mitochondrial membrane potential $\Delta \Psi_{\mathrm{m}}$ were analyzed with $5,5^{\prime}, 6,6^{\prime}$-tetrachloro-1, $1^{\prime}, 3,3^{\prime}$-tetraethylbenzimidazole-carbocyanine iodide (JC-1; Molecular Probes, Inc., Eugene, OR, USA). This cyanine dye accumulates in the mitochondrial matrix under the influence of the $\Delta \Psi_{\mathrm{m}}$ and forms $\mathrm{J}$ aggregates which have characteristic absorption and emission spectra. ${ }^{37}$ The JC-1 staining method is reported to provide more accurate estimates of $\Delta \Psi_{\mathrm{m}}$ than DiOC6(3). ${ }^{38}$ Untreated controls and cells treated with camptothecin $(10 \mu \mathrm{M})$ for $1,2,3,4,5$ and $6 \mathrm{~h}$ were incubated in $0.4 \mathrm{ml}$ of RPMI with $0.5 \mu \mathrm{M} \mathrm{JC}-1$ for $10 \mathrm{~min}$. As a positive control for reduction of $\Delta \Psi_{\mathrm{m}}$, control cells were treated with the uncoupling agent CCCP $(1 \mu \mathrm{M})$ before labeling with JC-1. Cell suspensions were prepared for flow cytometry and excitation of the carbocyanine dye (JC-1) was with an argon ion laser at $488 \mathrm{~nm}$ on a Becton Dickinson Vantage instrument. The emission wavelengths were collected at $530 \mathrm{~nm}$ for the green or low membrane potential and $585 \mathrm{~nm}$ for the red or high membrane potential. The software used for the data analysis was CELLQues (Becton-Dickinson Immunocytochemistry Systems, Mountainview, CA, USA). After gating out small-sized debris, 10000 events were collected for each analysis. Histograms of the ratio of the red to green fluorescence were used to analyze $\Delta \Psi_{\mathrm{m}}$.

\section{Electron microscopy ${ }^{39}$}

Jurkat cells were cultured in the absence or presence of $10 \mu \mathrm{M}$ camptothecin $\pm z$-VAD for $6 \mathrm{~h}$. The growth medium was removed by two washes with PBS and replaced by Karnofsky's reagent, consisting of $4 \%$ paraformaldehyde and $2 \%$ glutaraldehyde in $0.1 \mathrm{M}$ cacodylate buffer ( $\mathrm{pH}$ 7.4). After fixation for $30 \mathrm{~min}$ at room temperature, cells

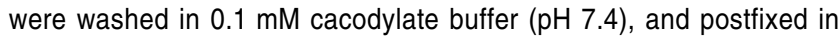
$2 \%$ osmium tetraoxide for $10 \mathrm{~min}$. Cells were dehydrated in an acetone series and embedded in Epon 812 . Thin $(60 \mathrm{~nm})$ sections were cut and placed on formvar-covered slot and mesh grids. The sections were stained with uranyl acetate and lead citrate. The samples were examined in a Philips 301 and a Zeiss 910 electron microscope.

\section{Statistical analysis}

All results are expressed as means \pm S.D. unless stated otherwise. The unpaired Student's $t$-test was used to evaluate the significance of differences between groups, accepting $P<0.05$ as a level of significance.

\section{Acknowledgements}

We thank Renji Song and Robert Dilwith for their help with the Flow Cytometry. This study was supported by $\mathrm{NIH}$ grants CA72455 and CA25933. We also acknowledge the videomicroscopy, cellular and molecular immunology, and EM core facilities of the Wadsworth Center, as well as Jan Galigan from the photography department for help with the figures.

\section{References}

1. Green DR and ReedJC (1998) Mitochondria and apoptosis. Science 281:1309 1312

2. Alnemri ES, Livingston DJ, Nicholson DW, Salvesen G, Thornberry NA, Wong WW and Yuan J (1996) Human ICE/CED-3 protease nomenclature. Cell 87: 171

3. Nicholson DW and Thornberry NA (1997) Caspases: killer proteases. Trends Biochem. Sci. 22: 299-306

4. Thornberry NA, Rosen A and Nicholson DW (1997) Control of apoptosis by proteases. Adv. Pharmacol. 41: 155-177

5. Stennicke HR and Salvesen GS (2000) Caspases controlling intracellular signals by protease zymogen activation. Biochim. Biophys. Acta 1477: 299-306

6. Lazebnik YA, Kaufmann SH, Desnoyers S, Poirier GG and Earnshaw WC (1994) Cleavage of poly(ADP-ribose) polymerase by a proteinase with properties like ICE. Nature 371: $346-347$

7. Thornberry NA and Lazebnik Y (1998) Caspases: enemies within. Science 281: $1312-1316$

8. Li P, Nijhawan D, Budihardjo I, Srinivasula SM, Ahmad M, Alnemri ES and Wang X (1997) Cytochrome $c$ and dATP-dependent formation of Apaf-1/caspase- 9 complex initiates an apoptotic protease cascade. Cell 91: 479-489

9. Vaux DL (1997) CED-4 - the third horseman of apoptosis. Cell 90: 389-390

10. Zou H, Henzel WJ, Liu X, Lutschg A and Wang X (1997) Apaf-1, a human protein homologous to $\mathrm{C}$. elegans CED-4, participates in cytochrome c-dependent activation of caspase-3. Cell 90: 405-413

11. Kluck RM, Bossy-Wetzel E, Green DR and Newmeyer DD (1997) The release of cytochrome c from mitochondria: a primary site for $\mathrm{Bcl}-2$ regulation of apoptosis. Science 275: 1132-1136

12. Kluck RM, Martin SJ, Hoffman BM, Zhou JS, Green DR and Newmeyer DD (1997) Cytochrome c activation of CPP32-like proteolysis plays a critical role in a Xenopus cell-free apoptosis system. EMBO J. 16: 4639-4649

13. Yang J, Liu X, BhallaK, Kim CN, Ibrado AM, Cai J, Peng TI, Jones DP and Wang X (1997) Prevention of apoptosis by Bcl-2: release of cytochrome c from mitochondria blocked. Science 275: 1129-1132

14. Liu X, Kim CN, Yang J, Jemmerson R and Wang X (1996) Induction of apoptotic program in cell-free extracts: requirement for dATP and cytochrome $c$. Cell 86: $147-157$

15. Samali A, Cai J, Zhivotovsky B, Jones DP and Orrenius S (1999) Presence of a pre-apoptotic complex of pro-caspase-3, Hsp60 and Hsp10 in the mitochondrial fraction of Jurkat cells. EMBO J. 18: 2040-2048

16. Mancini M, Nicholson DW, Roy S, Thornberry NA, Peterson EP, Casciola-Rosen LA and Rosen A (1998) The caspase-3 precursor has a cytosolic and mitochondrial distribution: implications for apoptotic signaling. J. Cell. Biol. 140: $1485-1595$

17. Xanthoudakis S, Roy S, Rasper D, Hennessy T, Aubin Y, Cassady R, Tawa P, Ruel R, Rosen A and Nicholson DW (1999) Hsp60 accelerates the maturation of pro-caspase-3 by upstream activator proteases during apoptosis. EMBO J. 18 : 2049-2056

18. Zhivotovsky B, Samali A, Gahm A and Orrenius S (1999) Caspases: their intracellular localization and translocation during apoptosis. Cell Death Differ. 6 : $644-651$

19. LiF, Srinivasan A, Wang Y, Armstrong RC, Tomaselli KJ and Fritz LC (1997) Cellspecific induction of apoptosis by microinjection of cytochrome $\mathrm{C}$. Bcl- $\mathrm{X}_{\mathrm{L}}$ has activity independent of cytochrome c release. J. Biol. Chem. 272: 30299-30305 
20. Vander Heiden MG, Chandel NS, Williamson EK, Schumacker PT and Thompson CB (1997) Bcl-xL regulates the membrane potential and volume homeostasis of mitochondria. Cell 91: 627-637

21. Banki K, Hutter E, Gonchoroff NJ and Perl A (1999) Elevation of mitochondria transmembrane potential and reactive oxygen intermediate levels are early events and occur independently from activation of caspases in Fas signaling. J. Immunol. 162: 1466-1479

22. Joshi B, Li L, Taffe BG, Zhu Z, Wahl S, Tian H, Ben-Josef E, Taylor JD, Porter AT and Tang DG (1999) Apoptosis induction by a novel anti-prostate cancer compound, BMD188 (a fatty acid-containing hydroxamic acid), requires the mitochondrial respiratory chain. Cancer Res. 59: 4343-4355

23. Skulachev VP (1998) Cytochrome $c$ in the apoptotic and antioxidant cascades. FEBS Lett. 423: 275-280

24. Turrens JF and Boveris A (1980) Generation of superoxide anion by the NADH dehydrogenase of bovine heart mitochondria. Biochem. J. 191: 421-427

25. Turrens JF, Alexandre A and Lehninger AL (1985) Ubisemiquinone is the electron donor for superoxide formation by complex III of heart mitochondria. Arch. Biochem. Biophys. 237: 408-414

26. Hatefi $Y$ (1985) The mitochondrial electron transport and oxidative phosphorylation system. Annu. Rev. Biochem. 54: 1015-1069

27. Goldstein JC, Waterhouse NJ, Juin P, Evan GI and Green DR (2000) The coordinate release of cytochrome $c$ during apoptosis is rapid, complete and kinetically invariant. Nature Cell Biol. 2: 156-162

28. SchulerM, Bossy-WetzelE, Goldstein JC, FitzgeraldP and Green DR(2000)p53 induces apoptosis by caspase activation through mitochondrial cytochrome $c$ release. J. Biol. Chem. 275: 7337-7342

29. LiX, Du L and Darzynkiewicz Z (2000) During apoptosis of HL-60 and U-937 cells caspases are activated independently of dissipation of mitochondrial electrochemical potential. Exp. Cell Res. 257: 290-297

30. Bossy-WetzelE, NewmeyerDD and Green DR(1998) Mitochondrial cytochrome c release in apoptosis occurs upstream of DEVD-specific caspase activation and independently of mitochondrial membrane depolarization. EMBO J. 17: $37-49$
31. Langer T (2000) AAA proteases: cellular machines for degrading membrane proteins. Trends Biochem. Sci. 25: 247-251

32. Patel S and Latterich M (1998) The AAA team: related ATPases with diverse functions. Trends Cell Biol. 8: 65-71

33. Steglich G, Neupert W and Langer T (1999) Prohibitins regulate membrane protein degradation by the m-AAA protease in mitochondria. Mol. Cell Biol. 19: 3435-3442

34. Arlt H, Steglich G, Perryman R, Guiard B, Neupert W and Langer T (1998) The formation of respiratory chain complexes in mitochondria is under the proteolytic control of the m-AAA protease. EMBO J. 17: 4837-4847

35. Krippner A, Matsuno-Yagi A, Gottlieb RA and Babior BM (1996) Loss of function of cytochrome $\mathrm{C}$ in Jurkat cells undergoing fas-mediated apoptosis. J. Biol. Chem. 271: 21629-21636

36. Sánchez-Alcázar JA, Schneider E, Martínez MA, Carmona P, HernándezMuñoz I, Siles E, De la Torre P, Ruíz-Cabello J, Garcia I and Solís-Herruzo JA (2000) Tumor Necrosis Factor- $\alpha$ increases the steady-state reduction of cytochrome $b$ of the mitochondrial respiratory chain in metabolically inhibited L929 cells. J. Biol. Chem. 275: 13353-13361

37. Smiley ST, Reers M, Mottola-Hartshorn C, Lin M, Chen A, Smith TW, Steele Jr GD and Chen LB (1991) Intracellular heterogeneity in mitochondrial membrane potentials revealed by a J-aggregate-forming lipophilic cation JC-1. Proc. Natl. Acad. Sci. U.S.A. 88: $3671-3675$

38. Salvioli S, Ardizzoni A, Franceschi C and Cossarizza A (1997) JC-1, but not DiOC6(3) or rhodamine 123, is a reliable fluorescent probe to assess delta psi changes in intact cells: implications for studies on mitochondrial functionality during apoptosis. FEBS Lett. 411: 77-82

39. Robinson $G$ (1997) Theory and practice of histological techniques. In: Bancroft JD, Stevens A, (eds). Electron microscopy. Edinburgh: Churchill Livingstone, pp. $326-370$ 